\title{
REVIEW
}

Open Access

\section{Effects of mesenchymal stromal cell- conditioned media on measures of lung structure and function: a systematic review and meta-analysis of preclinical studies}

Alvaro Moreira ${ }^{1 *}$ (D), Rija Naqvi ${ }^{1}$, Kristen Hall ${ }^{1}$, Chimobi Emukah, John Martinez ${ }^{1}$, Axel Moreira², Evan Dittmar ${ }^{1}$, Sarah Zoretic ${ }^{1}$, Mary Evans ${ }^{1}$, Delanie Moses ${ }^{1}$ and Shamimunisa Mustafa ${ }^{1}$

\begin{abstract}
Background: Lung disease is a leading cause of morbidity and mortality. A breach in the lung alveolar-epithelial barrier and impairment in lung function are hallmarks of acute and chronic pulmonary illness. This review is part two of our previous work. In part 1, we demonstrated that CdM is as effective as MSCs in modulating inflammation. Herein, we investigated the effects of mesenchymal stromal cell (MSC)-conditioned media (CdM) on (i) lung architecture/function in animal models mimicking human lung disease, and (ii) performed a head-to-head comparison of CdM to MSCs.
\end{abstract}

Methods: Adhering to the animal Systematic Review Centre for Laboratory animal Experimentation protocol, we conducted a search of English articles in five medical databases. Two independent investigators collected information regarding lung: alveolarization, vasculogenesis, permeability, histologic injury, compliance, and measures of right ventricular hypertrophy and right pulmonary pressure. Meta-analysis was performed to generate random effect size using standardized mean difference with 95\% confidence interval.

Results: A total of 29 studies met inclusion. Lung diseases included bronchopulmonary dysplasia, asthma, pulmonary hypertension, acute respiratory distress syndrome, chronic obstructive pulmonary disease, and pulmonary fibrosis. CdM improved all measures of lung structure and function. Moreover, no statistical difference was observed in any of the lung measures between MSCs and CdM.

Conclusions: In this meta-analysis of animal models recapitulating human lung disease, CdM improved lung structure and function and had an effect size comparable to MSCs.

Keywords: Conditioned media, Mesenchymal stem cell, Lung disease, Animal, Review

\footnotetext{
*Correspondence: MoreiraA@uthscsa.edu

'Department of Pediatrics, Division of Neonatology, University of Texas Health Science-San Antonio, San Antonio, TX 78229-3900, USA

Full list of author information is available at the end of the article
}

C C The Author(s). 2020 Open Access This article is licensed under a Creative Commons Attribution 4.0 International License, which permits use, sharing, adaptation, distribution and reproduction in any medium or format, as long as you give appropriate credit to the original author(s) and the source, provide a link to the Creative Commons licence, and indicate if changes were made. The images or other third party material in this article are included in the article's Creative Commons licence, unless indicated otherwise in a credit line to the material. If material is not included in the article's Creative Commons licence and your intended use is not permitted by statutory regulation or exceeds the permitted use, you will need to obtain permission directly from the copyright holder. To view a copy of this licence, visit http://creativecommons.org/licenses/by/4.0/. The Creative Commons Public Domain Dedication waiver (http://creativecommons.org/publicdomain/zero/1.0/) applies to the data made available in this article, unless otherwise stated in a credit line to the data. 


\section{Background}

Pulmonary illness is a leading cause of morbidity and mortality [1]. In children, acute respiratory exacerbations are a common reason for primary care visits and are often implicated in hospitalizations [2, 3]. Many of these pulmonary conditions result in impairments in lung function that may last into adulthood [4, 5]. Consequently, identifying novel therapies for lung disease is highly warranted.

A unifying theme in many lung diseases includes inflammation [6-8]. While some inflammation is necessary to combat new disease and for proper wound healing, chronic inflammation may result in altered lung structure and function. During an acute illness, current therapies focus on restoring lung function by abating inflammation [9-11]. For instance, glucocorticoids are the mainstay therapy for reducing inflammation during acute exacerbations of asthma [12]. More recently, mesenchymal stromal/stem cells (MSCs) have shown encouraging outcomes in animal models of lung inflammation [13-15].

MSCs are promising agents as they are easily harvested, can be rapidly expanded, and can secrete factors (exosomes, microvesicles, microRNA) known to reduce inflammation [16-18]. The "secretome" or "conditioned media" of MSCs is considered biologically active and can be easily collected from the surrounding fluid of propagating cells [19-21]. Remarkably, preclinical studies suggest MSC conditioned media (CdM) may be as restorative as the MSCs themselves [22, 23]. We supported this observation in a previous systematic review and meta-analysis demonstrating that CdM is as effective as MSCs in modulating inflammation [24].

This review is an extension of our previous work. In this review, we examined the effects of CdM on (i) lung architecture/function in animal models recapitulating lung disease and (ii) compare these findings to MSCs. Given that the therapeutic benefit of MSCs is attributed to a paracrine fashion, we believed $\mathrm{CdM}$ would have comparable effects to MSCs.

\section{Methods}

\section{Overview and literature search}

The methods in our review abide to those outlined by the Systematic Review Centre for Laboratory Animal Experimentation (SYRCLE) [25]. Our protocol was registered through the Collaborative Approach to MetaAnalysis and Review of Data from Experimental Studies (CAMARADES) [26]. Details are described in our previous publication.

We conducted a literature search in five databases using the following terms: mesenchymal stem cellconditioned media, lung disease, and animal. The last search was performed on March 17th, 2020. Three independent investigators evaluated titles and abstracts, followed by full-text review.

\section{Inclusion criteria and outcomes of interest}

We included studies administering MSC-CdM to animal models of acute lung injury or acute respiratory distress syndrome (ALI/ARDS), asthma, bronchopulmonary dysplasia (BPD), chronic obstructive pulmonary disease (COPD), cystic fibrosis (CF), pneumonia, pulmonary fibrosis (PF), and pulmonary hypertension (PH). Refer to Supplementary File 1 for the list of included studies.

\section{Outcomes of interest}

Measures of lung structure and/or function were our primary endpoint. Lung architecture and function were assessed under the following categories: alveolarization, vasculogenesis, right ventricular hypertrophy, fibrosis, permeability, pulmonary pressures, compliance, and lung injury. Although the pathogenesis of the included lung diseases are heterogeneous, we combined all processes irrespective of disease. This was conducted to obtain a scoping overview of the impact of $\mathrm{CdM}$ on biologic processes implicated in lung disease. Subsequently, we assessed lung structure/function by disease in our subgroup analysis. Excluded studies were those which did not provide data concerning our primary outcome of inflammation.

\section{Data extraction}

Three groups of investigators were used (ED and CE; RN and JM; ME, DM, and SM) to collect data. Uniformity of data was assessed by the primary author. This data included general study design, animal model characteristics, conditioned media characteristics, and outcomes of interest.

\section{Data analysis}

A random effects model was used to generate forest plots. A minimum of three studies were required for each outcome to proceed with a meta-analysis. The estimated effect size of CdM or MSC on lung architecture/ function was determined using standardized mean difference (SMD) with a 95\% confidence interval (CI). Statistical heterogeneity between studies was calculated using the $I^{2}$ metric, and funnel plots were used to examine publication bias. If more than six articles were included per outcome, we conducted a subgroup analysis for disease, animal species, and route and dose of CdM administration. All statistical analyses were performed in $\mathrm{R}$ version 3.6.2; packages used included dmetar, metafor, and meta. 


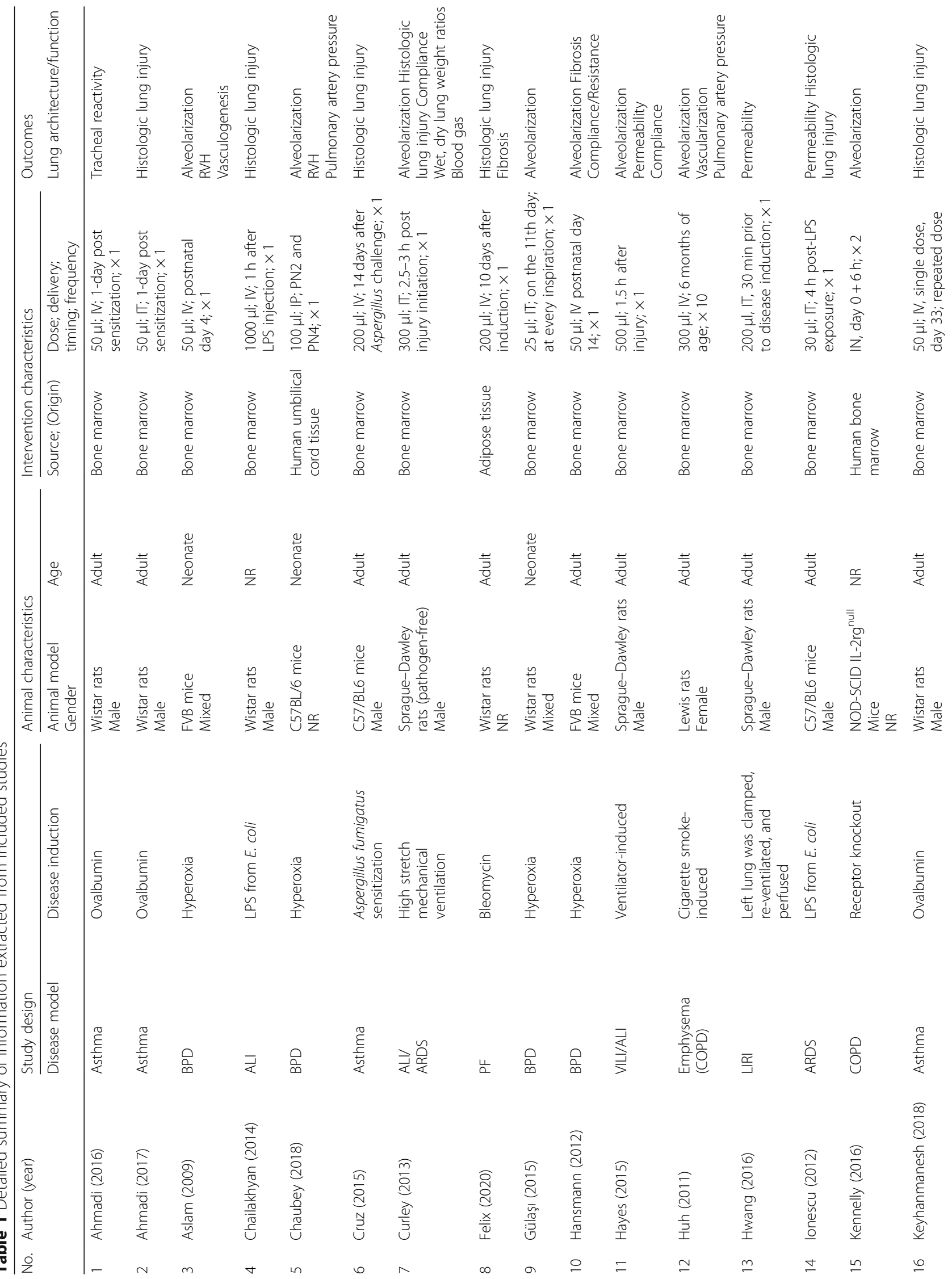




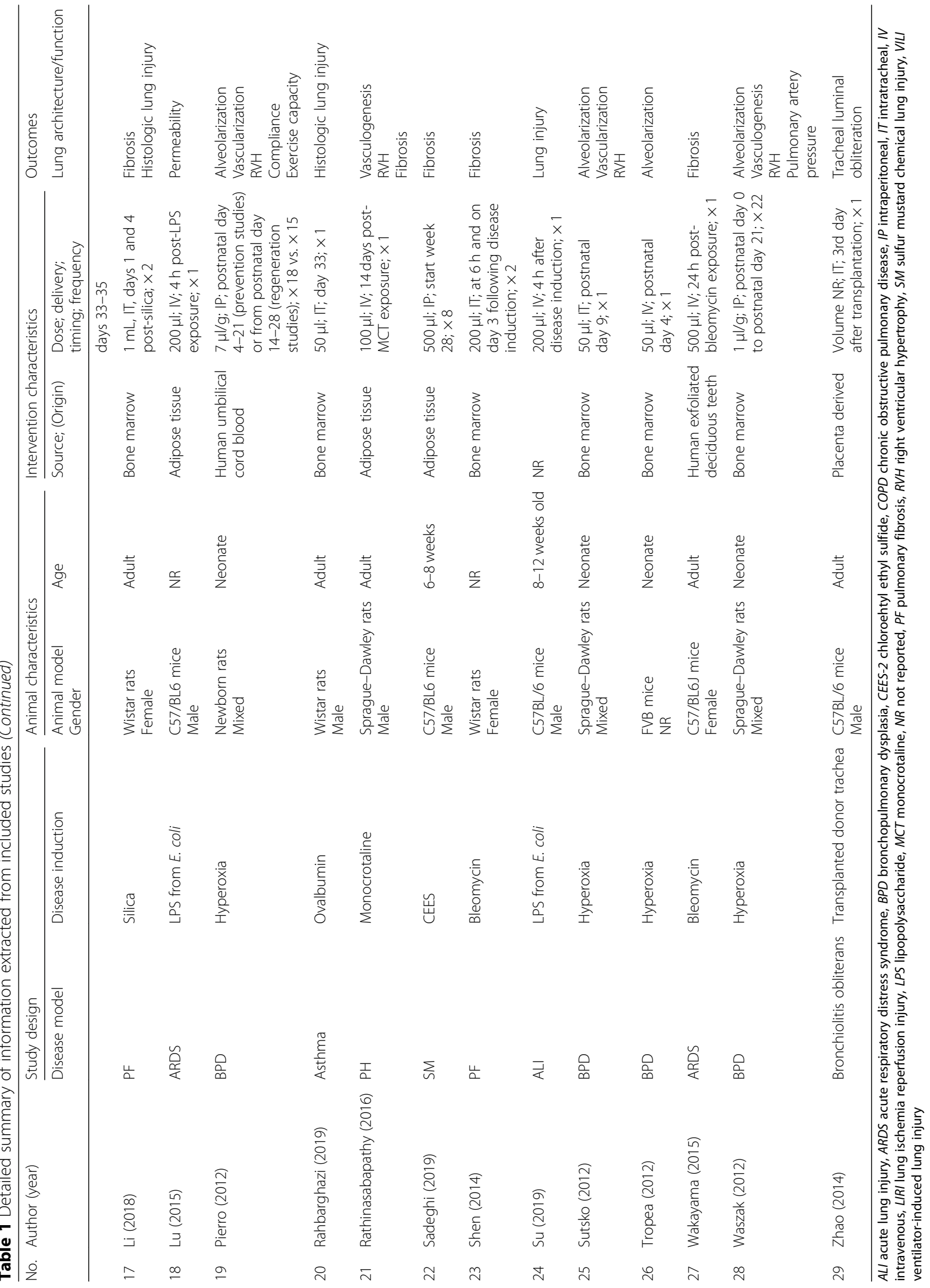




\section{Results}

\section{Study selection}

Our literature search resulted in 245 articles. After removing duplicates and viewing the titles and abstracts, 55 articles underwent full-text review. Twenty-nine articles met inclusion (refer to Supplementary Figure 1).

\section{Study details}

Table 1 summarizes the relevant study characteristics. Articles included in the review were published between the years 2009 to 2020 . BPD was the most common animal model $(n=8)$, followed by ALI/ARDS $(n=5)$ and asthma $(n=5)$. All of the studies used rodents to induce their lung model.

\section{CdM characteristics}

Conditioned media properties are summarized in Supplementary File 3. Stem cells were most isolated from bone marrows and cultured in Dulbecco's modified Eagle's medium. Incubation time of the $\mathrm{CdM}$ ranged from 24 to $72 \mathrm{~h}$. The volume of $\mathrm{CdM}$ administered ranged from $25 \mu \mathrm{l}$ to $1 \mathrm{ml}$.

\section{Alveolarization}

- CdM: improved alveolarization with an SMD of 1.32 (95\% CI 0.99, 1.65; 12 studies; Fig. 1a) with moderate heterogeneity $\left(I^{2}=67 \% ; p<0.01\right)$.

- MSC: improved alveolarization with an SMD of 1.80 (95\% CI 1.52, 2.07; 9 studies; Fig. 1b) with mild heterogeneity between groups $\left(I^{2}=36 \% ; p=0.01\right)$.

- $C d M$ vs. $M S C$ : no significant difference (Supplementary Figure 2).

\section{Right ventricular hypertrophy}

- CdM: favored CdM over control with an SMD of 1.08 (95\% CI - 1.56, - 0.61); 6 studies; Fig. 2a) with significant heterogeneity $\left(I^{2}=70 \% ; p<0.01\right)$.

- MSC: favored over the control with an SMD of 1.05 (95\% CI - 1.69, - 0.42; 3 studies, Fig. 2b) with significant heterogeneity between groups $\left(I^{2}=71 \%\right.$; $p<0.01)$.

- $C d M$ vs. $M S C$ : no significant difference (SMD 0.22, 95\% CI - 0.36, 0.16; Supplementary Figure 3).

\section{Lung fibrosis}

- CdM: favored CdM over control with an SMD of 1.08 (95\% CI - 1.56, - 0.61; 6 studies; Fig. 3a) with significant heterogeneity $\left(I^{2}=70 \% ; p<0.01\right)$.

- MSC: favored MSC over the control with an SMD of -1.99 (95\% CI - 2.93, - 1.04; 4 studies; Fig. 3b)

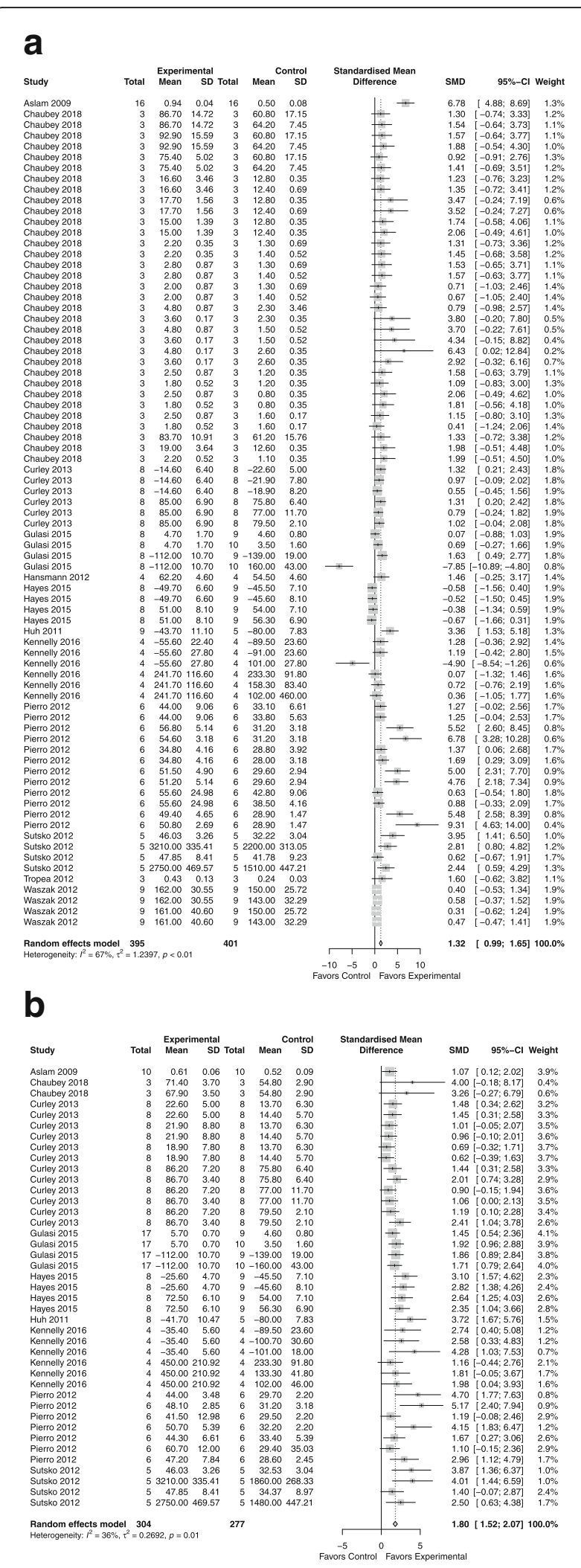

Fig. 1 Effect size of CdM (a) and MSC (b) on lung alveolarization. Forest plots demonstrate SMD with 95\% confidence interval 


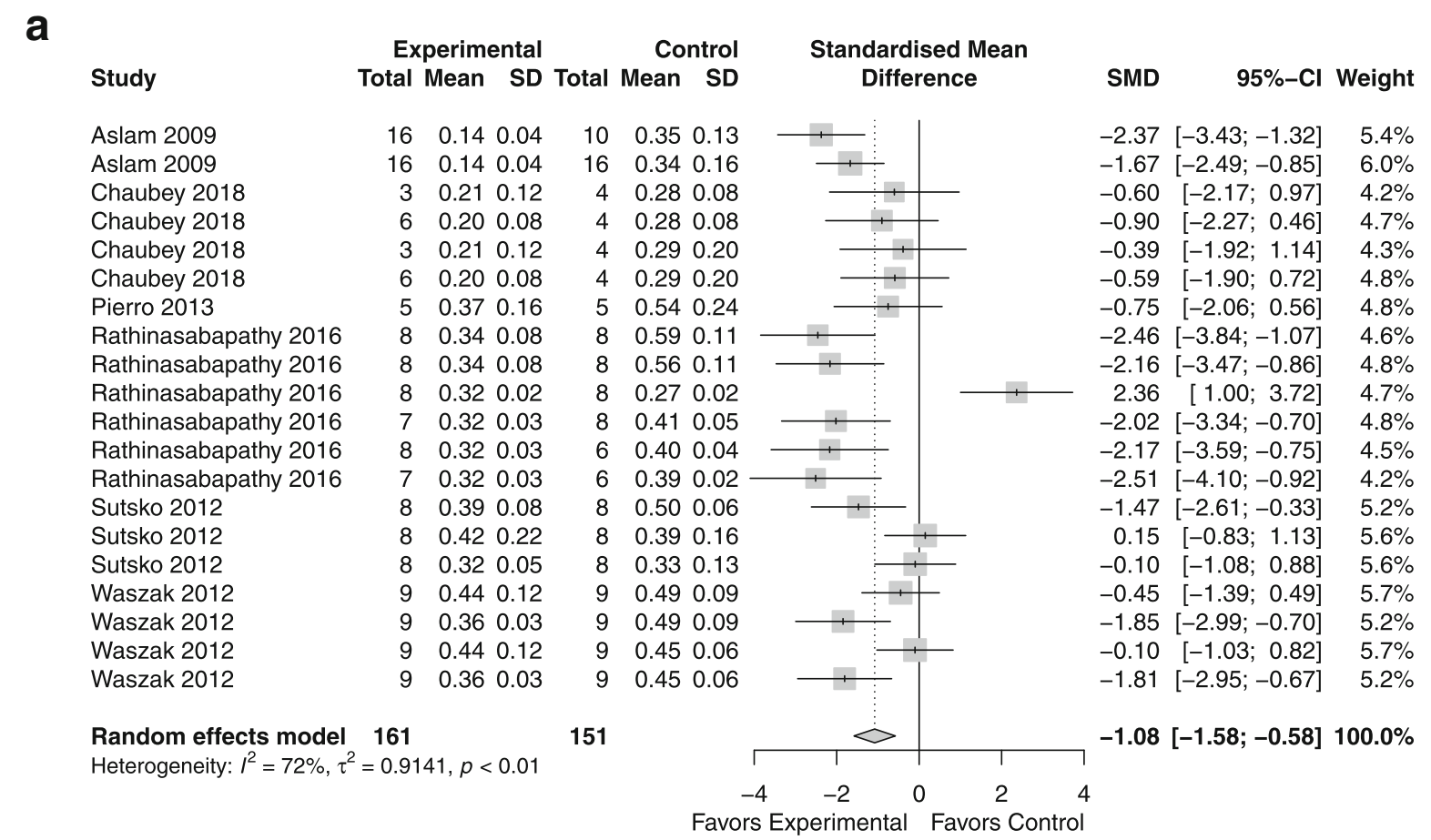

\section{b}

Study

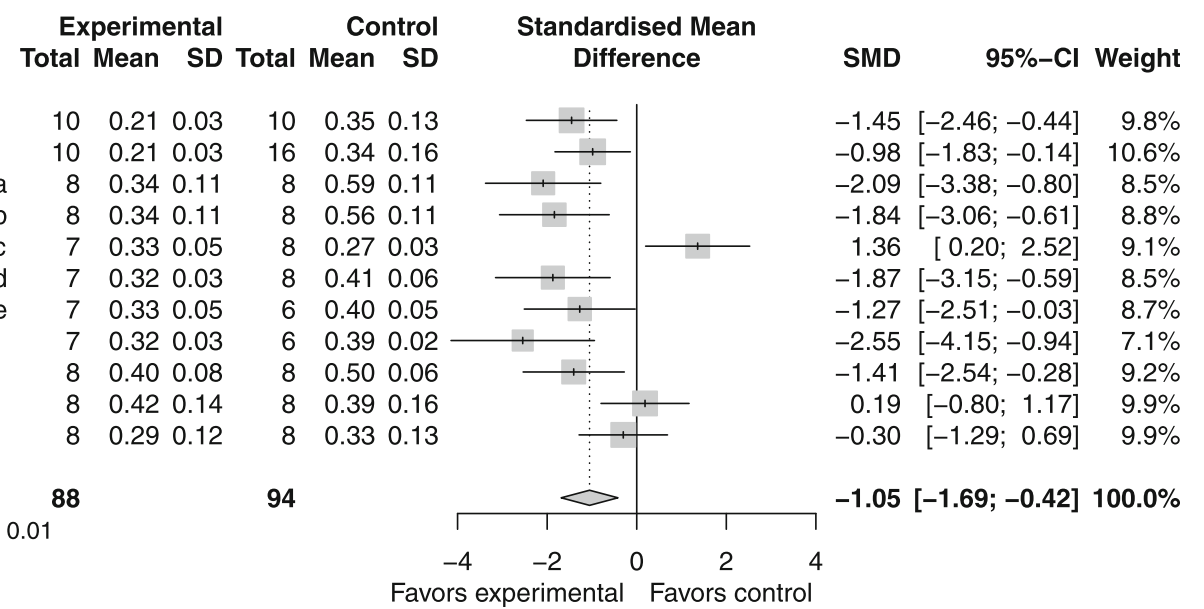

Fig. 2 Effect size of CdM (a) and MSC (b) on right ventricular hypertrophy. Forest plots demonstrate SMD with 95\% confidence interval

with significant heterogeneity between groups $\left(I^{2}=\right.$ 90\%; $p<0.01)$.

- $C d M$ vs. MSC: the comparison between CdM and MSCs was similar (refer to Supplementary Figure 4).

\section{Vasculogenesis}

- $C d M$ : superior to control with an SMD of -2.46 (95\% CI $-3.22,-1.70$; 6 studies; Fig. 4a) with moderate heterogeneity $\left(I^{2}=76 \% ; p<0.01\right)$.
- MSC: superior to control with an SMD of - 2.29 (95\% CI -3.01, - 1.56; 4 studies; Fig. 4b) with mild heterogeneity between groups $\left(I^{2}=35 \% ; p=0.14\right)$.

- $C d M$ vs. MSC: overall effectiveness between CdM and MSCs again showed no significant difference (Supplementary Figure 5).

\section{Permeability}

- $C d M$ : permeability assessment favored CdM over control with an SMD of - 0.99 (95\% CI - 1.32, - 
a

Study

Experimental Control Total Mean SD Total Mean SD

Standardised Mean Difference

Aslam 2009 Fulton a

Aslam 2009 Fulton b

Chaubey 2018 RV/LV +IVS a

Chaubey 2018 RV/LV a

Chaubey 2018 RV/LV +IVS b

Chaubey 2018 RV/LV b

Pierro 2013 Fulton

Rathinasabapathy 2016 RV/LV+IVS a

Rathinasabapathy 2016 RV/LV+IVS b

Rathinasabapathy 2016 RV/LV+IVS c

Rathinasabapathy 2016 RV/LV+IVS d

Rathinasabapathy 2016 RV/LV+IVS e

Rathinasabapathy 2016 RV/LV+IVS f

Sutsko 2012 RV/LV + IVS a

Sutsko 2012 RV/LV + IVS b

Sutsko 2012 RV/LV + IVS c

Waszak 2012 Fulton a

Waszak 2012 Fulton b

Waszak 2012 Fulton c

Waszak 2012 Fulton d

\section{Random effects model}

Heterogeneity: $I^{2}=70 \%, \tau^{2}=0.8031, p<0.01$

\section{$\begin{array}{lll}16 & 0.14 & 0.04\end{array}$ \\ $\begin{array}{llll}16 & 0.14 & 0.04\end{array}$ \\ $\begin{array}{llll}3 & 0.21 & 0.12\end{array}$ \\ $\begin{array}{lll}6 & 0.20 & 0.08\end{array}$ \\ $\begin{array}{llll}3 & 0.21 & 0.12\end{array}$ \\ $\begin{array}{lll}6 & 0.20 & 0.08\end{array}$ \\ $\begin{array}{llll}5 & 0.37 & 0.16\end{array}$ \\ $\begin{array}{llll}8 & 0.34 & 0.08\end{array}$ \\ $\begin{array}{llll}8 & 0.34 & 0.08\end{array}$ \\ $\begin{array}{llll}8 & 0.32 & 0.03\end{array}$ \\ $\begin{array}{lll}7 & 0.32 & 0.03\end{array}$ \\ $\begin{array}{llll}8 & 0.32 & 0.03\end{array}$ \\ $\begin{array}{llll}7 & 0.32 & 0.03\end{array}$ \\ $\begin{array}{llll}8 & 0.39 & 0.08\end{array}$ \\ $\begin{array}{llll}8 & 0.42 & 0.22\end{array}$ \\ $\begin{array}{llll}8 & 0.32 & 0.05\end{array}$ \\ $\begin{array}{llll}9 & 0.44 & 0.12\end{array}$ \\ $\begin{array}{llll}9 & 0.36 & 0.03\end{array}$ \\ $\begin{array}{llll}9 & 0.44 & 0.12\end{array}$ \\ $\begin{array}{lll}9 & 0.36 & 0.03\end{array}$}

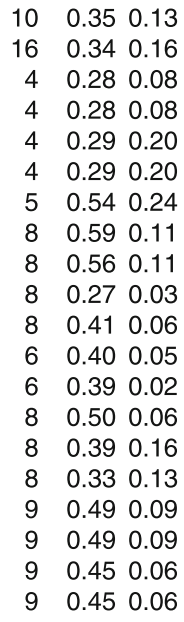

151

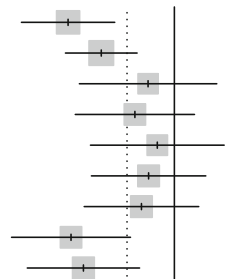

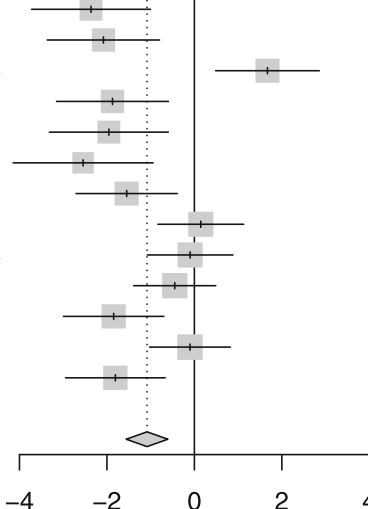

SMD $\quad 95 \%-C l$ Weight

$-2.43[-3.50 ;-1.36]$

$-1.67[-2.49 ;-0.85] \quad 6.1 \%$

$-0.60[-2.17 ; 0.97] \quad 4.1 \%$

$-0.90 \quad[-2.27 ; 0.46] \quad 4.6 \%$

$-0.39 \quad[-1.92 ; 1.14] \quad 4.2 \%$

-0.59 [-1.90; 0.72$] \quad 4.7 \%$

$-0.75[-2.06 ; 0.56] \quad 4.7 \%$

$-2.36[-3.72 ;-1.00] \quad 4.6 \%$

$-2.08[-3.36 ;-0.80] \quad 4.8 \%$

$1.67 \quad[0.49 ; 2.86] \quad 5.1 \%$

$-1.87[-3.15 ;-0.59] \quad 4.8 \%$

$-1.96[-3.32 ;-0.60] \quad 4.6 \%$

$-2.55[-4.15 ;-0.94] \quad 4.0 \%$

$-1.55[-2.71 ;-0.39] \quad 5.1 \%$

$0.15[-0.83 ; 1.13] \quad 5.6 \%$

$-0.10 \quad[-1.08 ; 0.88] \quad 5.6 \%$

$-0.45[-1.39 ; 0.49] \quad 5.7 \%$

$-1.85[-2.99 ;-0.70] \quad 5.2 \%$

$-0.10 \quad[-1.03 ; 0.82] \quad 5.8 \%$

$-1.81[-2.95 ;-0.67] \quad 5.2 \%$

$-1.08[-1.56 ;-0.61] 100.0 \%$

Favors Experimental Favors Control

b

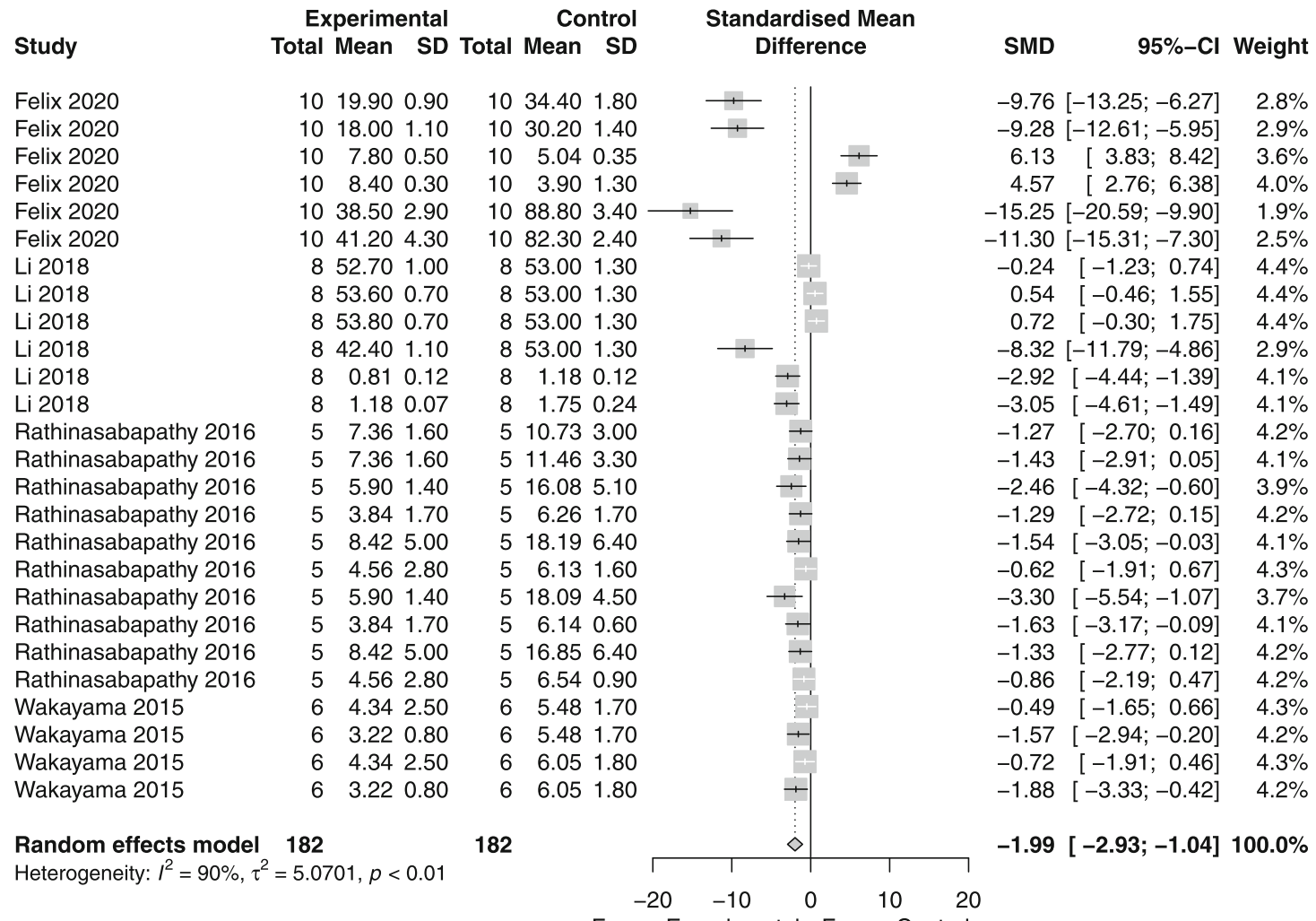

Favors Experimental Favors Control

Fig. 3 Effect size of CdM (a) and MSC (b) on lung fibrosis. Forest plots demonstrate SMD with 95\% confidence interval 


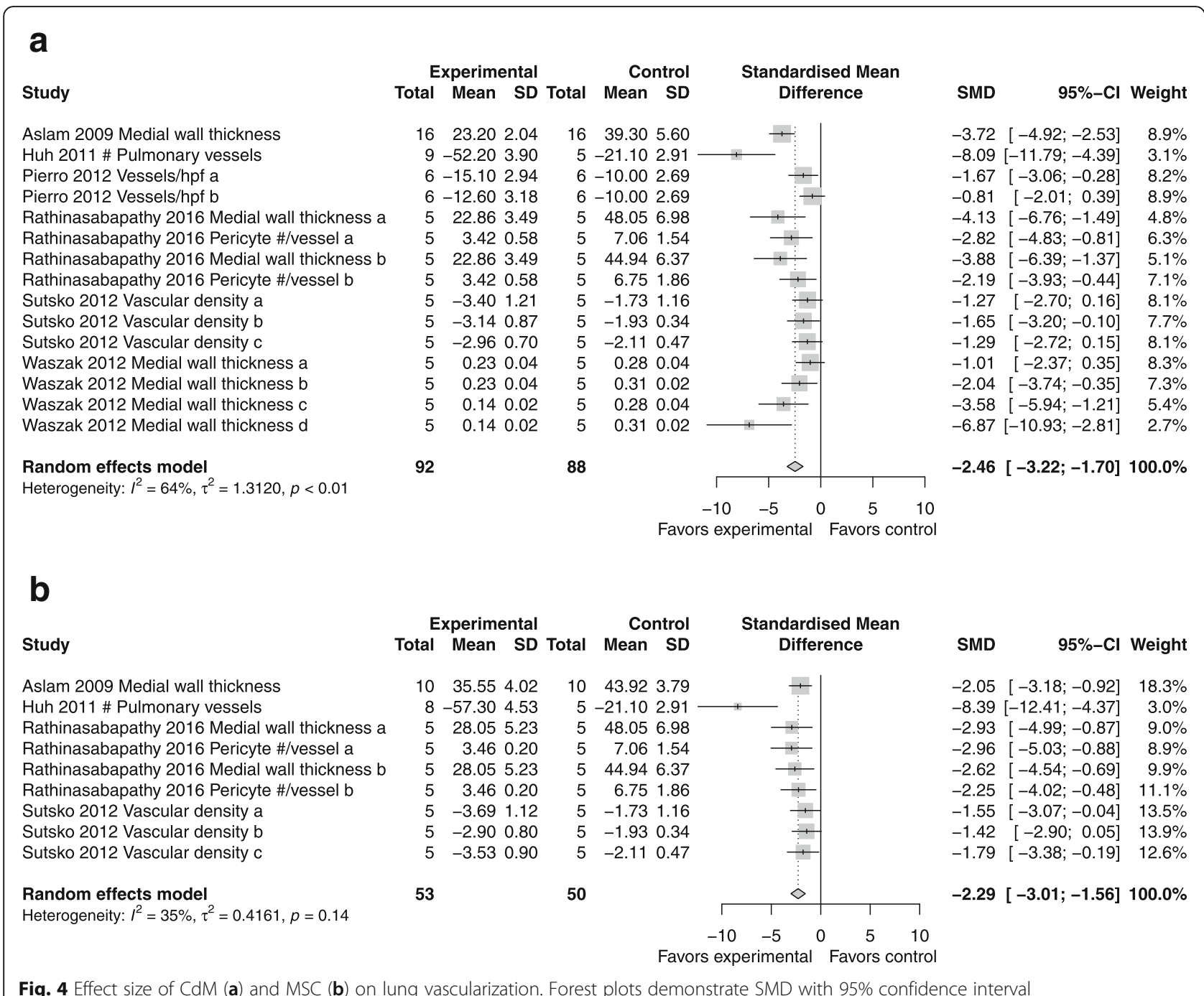

0.66; 5 studies; Fig. 5a) homogeneity that is nonsignificant $\left(I^{2}=11.0 \% ; p=0.33\right)$.

- MSC: in the evaluation of permeability, the MSC was favored over the control with an effect size of 1.54 (95\% CI -2.13, - 0.95; 4 studies; Fig. 5b) with heterogeneity between groups $\left(I^{2}=57.0 \%\right.$; $\left.p<0.01\right)$.

- $C d M$ vs. $M S C$ : equal effectiveness (Supplementary Figure 6).

\section{Pulmonary pressures}

- $C d M$ : improvement in right ventricular pressures compared to control with an SMD of - 0.69 (95\% CI $-0.99,-0.39$; 5 studies; Fig. 6a) with moderate heterogeneity $\left(I^{2}=51 \% ; p<0.01\right)$.

- MSC: superior to control with an SMD of - 1.63 (95\% CI - 2.02, - 1.24; 3 studies; Fig. 6b) with moderate heterogeneity $\left(I^{2}=63 \% ; p<0.01\right)$.
- $C d M$ vs. $M S C$ : comparable (please refer to Supplementary Figure 7).

\section{Histologic lung injury}

- $C d M$ : improvement in histologic lung injury compared to control with an SMD of - 6.05 (95\% CI $-8.72,-3.38$; 3 studies; Fig. 7a) with significant heterogeneity $\left(I^{2}=87 \% ; p<0.01\right)$.

- MSC: superior to control with an SMD of - 2.01 (95\% CI -3.41, - 0.60; 3 studies; Fig. 7b) with significant heterogeneity $\left(I^{2}=88 \% ; p<0.01\right)$.

- $C d M$ vs. MSC: less than 3 studies; comparison not performed.

\section{Compliance}

- CdM: improvement in lung compliance compared to control with an SMD of 1.75 (95\% CI 0.81, 2.69; 4 


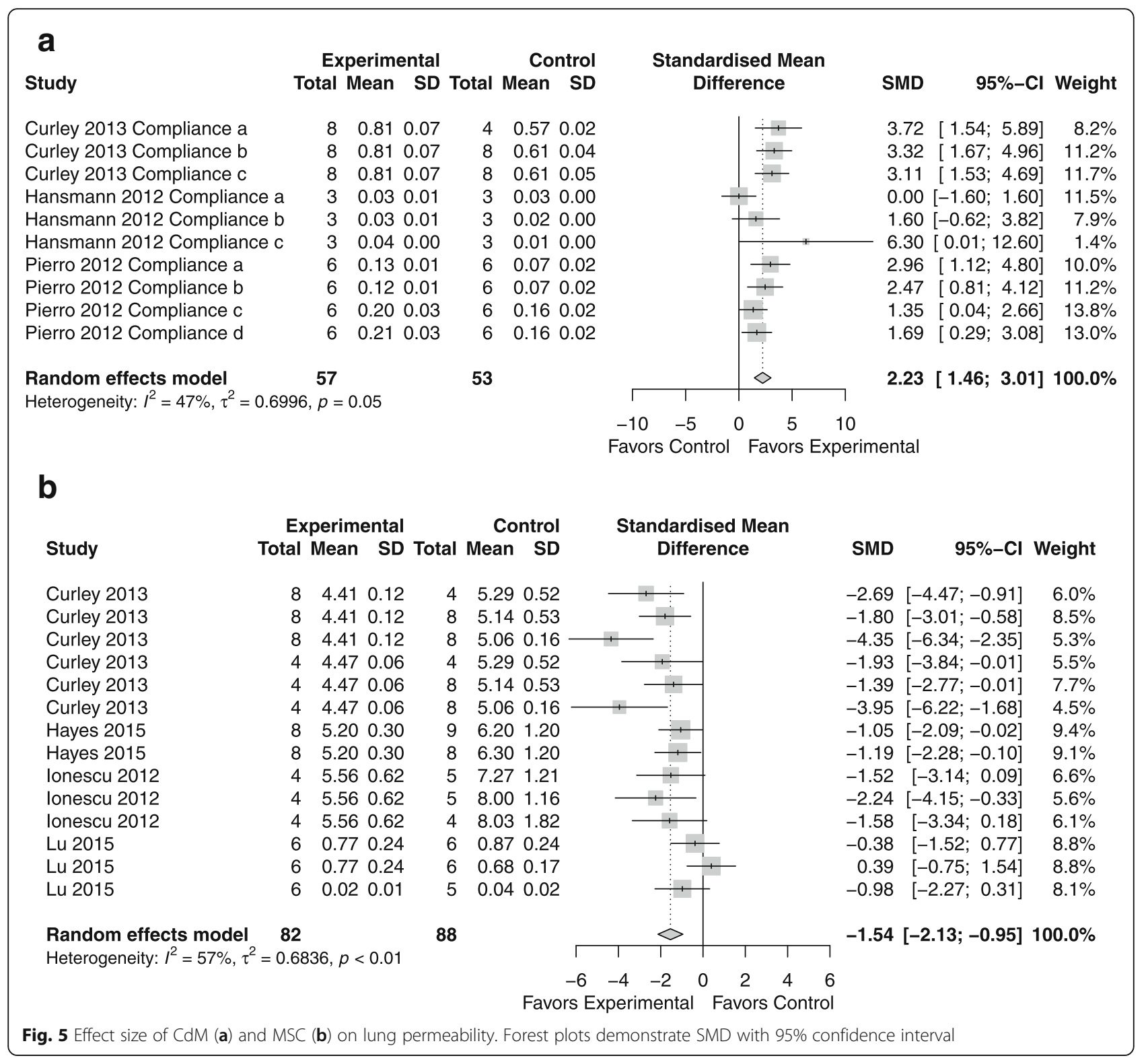

studies; Fig. 8a) with significant heterogeneity $\left(I^{2}=\right.$ 76\%; $\mathrm{p}<0.01)$.

- $M S C$ : improvement in lung compliance compared to control with an SMD of 2.33 (95\% CI 1.84, 2.82; 3 studies; Fig. $8 \mathrm{~b})$ with no heterogeneity $\left(I^{2}=0 \%\right.$; $p=0.5)$.

- $C d M$ vs. $M S C$ : not applicable as less than three studies performed a head-to-head comparison.

\section{All outcomes for lung structure and function combined}

- $C d M$ : Supplementary Figure 8A shows the SMD of -1.38 (with $95 \%$ CI of $-1.57,-1.19$ ) favoring CdM over control.
- MSC: Supplementary Figure 8B shows the SMD of -1.66 (with $95 \%$ CI of $-1.91,-1.41$ ) favoring MSC over control.

- $C d M$ vs. $M S C$ : no difference was appreciated between CdM and MSC when all outcomes were combined (Supplementary Figure 8C).

\section{Subgroup analysis}

Stratification of data was performed by lung disease, tissue source, dose, and route of delivery of CdM. Evaluation was performed if more than 6 studies had data.

\section{Alveolarization}

Supplementary Figure 9A-D demonstrates that CdM had the greatest impact on alveolarization in BPD 
a Study

Chaubey 2018 PAAT a

Chaubey 2018 PAAT b

Chaubey 2018 PAAT c

Chaubey 2018 PAAT d

Chaubey 2018 PAAT/PAET a

Chaubey 2018 PAAT/PAET b

Chaubey 2018 PAAT/PAET c

Chaubey 2018 PAAT/PAET d

Chaubey 2018 PAAT/PAET e

Chaubey 2018 PAAT/PAET $f$

Huh 2011 RVSP

Sutsko 2012 RVSP a

Sutsko 2012 RVSP b

Sutsko 2012 RVSP c

Rathinasabapathy 2016 RVSP a

Rathinasabapathy 2016 RVEDP a

Rathinasabapathy 2016 RV/LV EDA a

Rathinasabapathy 2016 RV/LV EF a

Rathinasabapathy 2016 At/Et a

Rathinasabapathy 2016 RVOT vmax a

Rathinasabapathy 2016 RVSP b

Rathinasabapathy 2016 RVEDP b

Rathinasabapathy 2016 RV/LV EDA b

Rathinasabapathy 2016 RV/LV EF b

Rathinasabapathy $2016 \mathrm{At} / \mathrm{Et} \mathrm{b}$

Rathinasabapathy 2016 RVOT vmax b

Rathinasabapathy 2016 RVSP c

Waszak 2012 PAAT/RVET a

Waszak 2013 PAAT/RVET b

Waszak 2014 PAAT/RVET c

Waszak 2015 PAAT/RVET d

Random effects model

Heterogeneity: $I^{2}=51 \%, \tau^{2}=0.3589, p<0.01$

\begin{tabular}{|c|c|c|c|c|c|c|c|}
\hline \multicolumn{4}{|c|}{ Experimental } & \multicolumn{2}{|c|}{ Control } & \multirow{2}{*}{\multicolumn{2}{|c|}{$\begin{array}{l}\text { Standardised Mean } \\
\text { Difference }\end{array}$}} \\
\hline Total & Mean & SD & Total & Mean & SD & & \\
\hline & -24.90 & 12.52 & & -21.80 & 6.71 & & \\
\hline & -33.80 & 25.94 & & -21.80 & 6.71 & & — \\
\hline & -24.90 & 12.52 & & -20.90 & 20.12 & & \\
\hline & -33.80 & 25.94 & & -20.90 & 20.12 & & E \\
\hline 3 & -0.32 & 0.09 & 4 & -0.21 & 0.09 & 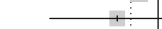 & 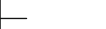 \\
\hline 3 & -0.32 & 0.09 & 4 & -0.22 & 0.27 & 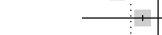 & \\
\hline 3 & -0.31 & 0.18 & 4 & -0.21 & 0.13 & 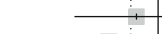 & - \\
\hline 6 & -0.36 & 0.09 & 4 & -0.21 & 0.13 & 4 & \\
\hline 3 & -0.31 & 0.18 & 4 & -0.22 & 0.27 & & \\
\hline 6 & -0.36 & 0.09 & 4 & -0.22 & 0.27 & & - \\
\hline 9 & 23.00 & 8.05 & 5 & 29.30 & 9.39 & i & - \\
\hline 20 & 23.43 & 3.49 & 20 & 33.35 & 3.49 & $\longrightarrow$ & \\
\hline 10 & 37.48 & 19.86 & 10 & 40.30 & 20.80 & & \\
\hline 8 & 38.89 & 9.35 & 8 & 41.57 & 9.79 & & \\
\hline 8 & 55.09 & 26.79 & 8 & 82.64 & 21.42 & 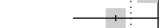 & \\
\hline 8 & 7.37 & 4.34 & 8 & 12.80 & 4.02 & + & \\
\hline 8 & 0.59 & 0.27 & 8 & 2.03 & 1.25 & 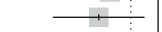 & \\
\hline 8 & -0.56 & 0.18 & 8 & -0.37 & 0.27 & $\div$ & \\
\hline 8 & -0.30 & 0.04 & 8 & -0.37 & 0.04 & & - \\
\hline 8 & -0.76 & 0.09 & 8 & -0.87 & 0.13 & & 1 \\
\hline 8 & 55.09 & 26.79 & 8 & 79.04 & 26.79 & $\ldots$ & \\
\hline 8 & 7.37 & 4.34 & 8 & 11.70 & 2.91 & 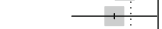 & \\
\hline 8 & 0.59 & 0.27 & 5 & 1.91 & 1.65 & 1 & \\
\hline 8 & -0.56 & 0.18 & 5 & -0.35 & 0.13 & 1 & \\
\hline 8 & -0.30 & 0.04 & 5 & -0.19 & 0.04 & $+\quad$ & \\
\hline 8 & -0.76 & 0.09 & 5 & -0.55 & 0.22 & 4 & \\
\hline 8 & 34.84 & 17.62 & 8 & 45.55 & 11.14 & $\rightarrow$ & \\
\hline 9 & -0.20 & 0.13 & 9 & -0.18 & 0.04 & & \\
\hline 9 & -0.23 & 0.09 & 9 & -0.18 & 0.04 & $-i$ & \\
\hline 9 & -0.20 & 0.13 & 9 & -0.19 & 0.09 & & \\
\hline 9 & -0.23 & 0.09 & 9 & -0.19 & 0.09 & & \\
\hline 229 & & & 211 & & & & \\
\hline 0.01 & & & & & & \begin{tabular}{ccc|} 
& 1 & \\
-3 & -2 & -1
\end{tabular} & $\begin{array}{ll}1 & 1 \\
1 & 2\end{array}$ \\
\hline
\end{tabular}

Favors experimental Favors control
SMD $\quad 95 \%-C l$ Weight

$-0.28 \quad[-1.79 ; 1.24] \quad 2.4 \%$

$-0.52[-1.82 ; 0.78] \quad 2.9 \%$

$-0.19[-1.70 ; 1.31] \quad 2.5 \%$

$-0.49[-1.78 ; 0.81] \quad 2.9 \%$

$-1.04[-2.74 ; 0.67] \quad 2.1 \%$

$-0.39[-1.92 ; 1.14] \quad 2.4 \%$

$-0.55[-2.11 ; 1.01] \quad 2.3 \%$

$-1.25[-2.70 ; 0.20] \quad 2.6 \%$

$-0.32[-1.84 ; 1.20] \quad 2.4 \%$

$-0.71[-2.03 ; 0.62] \quad 2.8 \%$

$-0.69[-1.83 ; 0.44] \quad 3.4 \%$

$-2.79[-3.68 ;-1.89] \quad 4.1 \%$

$-0.13[-1.01 ; 0.74] \quad 4.2 \%$

$-0.26[-1.25 ; 0.72] \quad 3.8 \%$

$-1.07[-2.14 ; 0.00] \quad 3.5 \%$

$-1.23[-2.32 ;-0.13] \quad 3.5 \%$

$-1.50[-2.65 ;-0.35] \quad 3.3 \%$

$-0.79[-1.82 ; 0.24] \quad 3.7 \%$

$1.48 \quad[0.34 ; 2.62] \quad 3.3 \%$

$0.91[-0.13 ; 1.96] \quad 3.6 \%$

$-0.85[-1.88 ; 0.19] \quad 3.6 \%$

$-1.11[-2.18 ;-0.03] \quad 3.5 \%$

$-1.20[-2.45 ; 0.04] \quad 3.0 \%$

$-1.19 \quad[-2.44 ; 0.05] \quad 3.1 \%$

$-2.29[-3.82 ;-0.75] \quad 2.4 \%$

$-1.28[-2.54 ;-0.02] \quad 3.0 \%$

$-0.69[-1.70 ; 0.33] \quad 3.7 \%$

$-0.19[-1.12 ; 0.74] \quad 4.0 \%$

$-0.67[-1.63 ; 0.28]-3.9 \%$

$-0.08[-1.01 ; 0.84]-4.0 \%$

$-0.43[-1.36 ; 0.51] \quad 4.0 \%$

$-0.69[-0.99 ;-0.39] 100.0 \%$

\section{b}

Study

Huh 2011 RVSP

Huh 2011 MPAP

Rathinasabapathy 2016 RVSP a

Rathinasabapathy 2016 RVEDP a

Rathinasabapathy 2016 RV/LV EDA a

Rathinasabapathy 2016 RV/LV EF a

Rathinasabapathy 2016 At/Et a

Rathinasabapathy 2016 RVOT vmax a

Rathinasabapathy 2016 RVSP b

Rathinasabapathy 2016 RVEDP b

Rathinasabapathy 2016 RV/LV EDA b

Rathinasabapathy 2016 RV/LV EF b

Rathinasabapathy $2016 \mathrm{At} / \mathrm{Et} \mathrm{b}$

Rathinasabapathy 2016 RVOT vmax b

Rathinasabapathy 2016 RVSP c

Rathinasabapathy 2016 RV/LV EDA c

Rathinasabapathy 2016 RV/LV EF c

Rathinasabapathy 2016 RVSP d

Rathinasabapathy 2016 RV/LV EDA d

Rathinasabapathy 2016 RV/LV EF d

Rathinasabapathy 2016 RVSP

Rathinasabapathy 2016 RV/LV EDA e

Rathinasabapathy 2016 RV/LV EF e

Rathinasabapathy 2016 RVSP $f$

Rathinasabapathy 2016 RV/LV EDA f

Rathinasabapathy 2016 RV/LV EF f

Sutsko 2012 RVSP a

Sutsko 2012 RVSP b

Sutsko 2012 RVSP c

Random effects model

Heterogeneity: $I^{2}=63 \%, \tau^{2}=0.6913, p<0.01$
Experimental Control Standardised Mean

Difference

$\begin{array}{lll}8 & 17.60 & 5.37\end{array}$

$\begin{array}{lll}8 & 15.60 & 2.26\end{array}$

$\begin{array}{llll}8 & 58.08 & 13.58\end{array}$

$\begin{array}{lll}8 & 7.63 & 2.38\end{array}$

$\begin{array}{lll}8 & 0.68 & 0.48\end{array}$

$\begin{array}{lll}8 & -0.63 & 0.08\end{array}$

$\begin{array}{lll}8 & -0.33 & 0.06\end{array}$

$\begin{array}{llll}8 & -0.74 & 0.08\end{array}$

$\begin{array}{llll}8 & 58.08 & 13.58\end{array}$

$\begin{array}{lll}8 & 7.63 & 2.38\end{array}$

$\begin{array}{llll}8 & 0.68 & 0.48\end{array}$

$\begin{array}{lll}8 & -0.63 & 0.08\end{array}$

$\begin{array}{lll}8 & -0.33 & 0.06\end{array}$

$\begin{array}{lll}8 & -0.74 & 0.08\end{array}$

$\begin{array}{lll}7 & 33.29 & 4.21\end{array}$

$\begin{array}{lll}7 & 0.38 & 0.05\end{array}$

$\begin{array}{lll}7 & -0.65 & 0.08\end{array}$

$\begin{array}{llll}7 & 36.22 & 7.41\end{array}$

$\begin{array}{lll}7 & 0.37 & 0.05\end{array}$

$\begin{array}{lll}7 & -0.69 & 0.11\end{array}$

$\begin{array}{lll}7 & 33.29 & 4.21\end{array}$

$\begin{array}{lll}7 & 0.38 & 0.05\end{array}$

$\begin{array}{lll}7 & -0.65 & 0.08\end{array}$

$\begin{array}{lll}7 & 36.22 & 7.41\end{array}$

$\begin{array}{lll}7 & 0.37 & 0.05\end{array}$

$\begin{array}{lll}7 & -0.69 & 0.11\end{array}$

$\begin{array}{lll}20 & 25.80 & 3.49\end{array}$

1029.7018 .72

$\begin{array}{lll}8 & 28.81 \quad 5.88\end{array}$

234

216 $\begin{array}{lll}5 & 29.30 \quad 4.70\end{array}$

$\begin{array}{lll}5 & 21.10 \quad 3.80\end{array}$

882.6413 .55

$\begin{array}{lll}8 & 12.80 & 2.55\end{array}$

$\begin{array}{llll}8 & 2.03 & 0.79\end{array}$

$\begin{array}{lll}8 & -0.37 & 0.17\end{array}$

$\begin{array}{lll}8 & -0.37 & 0.03\end{array}$

$\begin{array}{llll}8 & -0.87 & 0.08\end{array}$

$\begin{array}{llll}8 & 79.04 & 16.94\end{array}$

$\begin{array}{lll}8 & 11.70 & 1.84\end{array}$

$\begin{array}{lll}5 & 1.91 & 0.83\end{array}$

$\begin{array}{lll}5 & -0.35 & 0.07\end{array}$

$\begin{array}{lll}5 & -0.19 & 0.02\end{array}$

$\begin{array}{lll}5 & -0.55 & 0.11\end{array}$

$\begin{array}{llll}8 & 45.55 & 7.04\end{array}$

$\begin{array}{lll}8 & 0.64 & 0.14\end{array}$

$\begin{array}{lll}8 & -0.53 & 0.11\end{array}$

$8 \quad 45.71 \quad 11.09$

$\begin{array}{lll}8 & 0.71 & 0.11\end{array}$

$\begin{array}{lll}8 & -0.56 & 0.08\end{array}$

$\begin{array}{lll}6 & 46.98 & 6.91\end{array}$

$\begin{array}{lll}6 & 0.68 & 0.12\end{array}$

$\begin{array}{lll}6 & -0.54 & 0.07\end{array}$

$\begin{array}{lll}6 & 48.03 \quad 6.76\end{array}$

$\begin{array}{lll}6 & 0.65 & 0.12\end{array}$

$\begin{array}{lll}6 & -0.53 & 0.07\end{array}$

$\begin{array}{lll}20 & 33.35 & 3.49\end{array}$

$\begin{array}{llll}10 & 40.30 & 14.70\end{array}$

$\begin{array}{lll}8 & 41.57 \quad 6.19\end{array}$

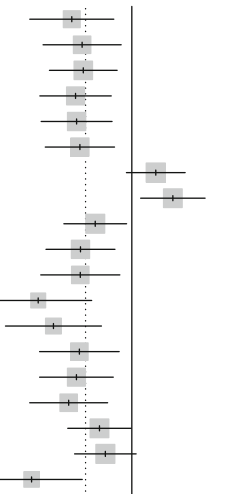

SMD $\quad 95 \%-\mathrm{Cl}$ Weight

$-2.12[-3.60 ;-0.64] \quad 3.1 \%$

$-1.75[-3.13 ;-0.38] \quad 3.3 \%$

$-1.71[-2.91 ;-0.52] \quad 3.7 \%$

$-1.99[-3.25 ;-0.73] \quad 3.5 \%$

$-1.95[-3.20 ;-0.70] \quad 3.6 \%$

$-1.83[-3.05 ;-0.61] \quad 3.6 \%$

$0.85 \quad[-0.19 ; 1.88] \quad 4.0 \%$

$1.45 \quad[0.31 ; 2.59] \quad 3.8 \%$

$-1.29[-2.40 ;-0.18] \quad 3.9 \%$

$-1.81[-3.03 ;-0.59] \quad 3.6 \%$

$-1.82[-3.21 ;-0.42] \quad 3.3 \%$

$-3.30[-5.19 ;-1.42] \quad 2.4 \%$

$-2.77[-4.46 ;-1.07] \quad 2.7 \%$

$-1.85[-3.26 ;-0.45] \quad 3.3 \%$

$-1.95[-3.25 ;-0.65] \quad 3.5 \%$

$-2.23[-3.60 ;-0.85] \quad 3.3 \%$

$-1.14[-2.26 ;-0.02] \quad 3.8 \%$

$-0.93[-2.02 ; 0.15] \quad 3.9 \%$

$-3.54[-5.33 ;-1.75] \quad 2.6 \%$

$-1.29[-2.43 ;-0.14] \quad 3.8 \%$

$-2.27[-3.79 ;-0.76] \quad 3.0 \%$

$-3.05[-4.83 ;-1.28] \quad 2.6 \%$

$-1.33[-2.58 ;-0.08] \quad 3.6 \%$

$-1.54[-2.84 ;-0.24] \quad 3.5 \%$

$-2.85[-4.56 ;-1.14] \quad 2.7 \%$

$-1.61[-2.93 ;-0.29] \quad 3.4 \%$

$-2.12[-2.91 ;-1.33] \quad 4.6 \%$

$-0.60[-1.50 ; 0.30] \quad 4.3 \%$

$-2.00[-3.26 ;-0.73] \quad 3.5 \%$

$-1.63[-2.02 ;-1.24] 100.0 \%$

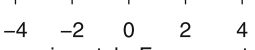

Favors experimental Favors contro

Fig. 6 Effect size of CdM (a) and MSC (b) on pulmonary pressures. Forest plots demonstrate SMD with 95\% confidence interval 


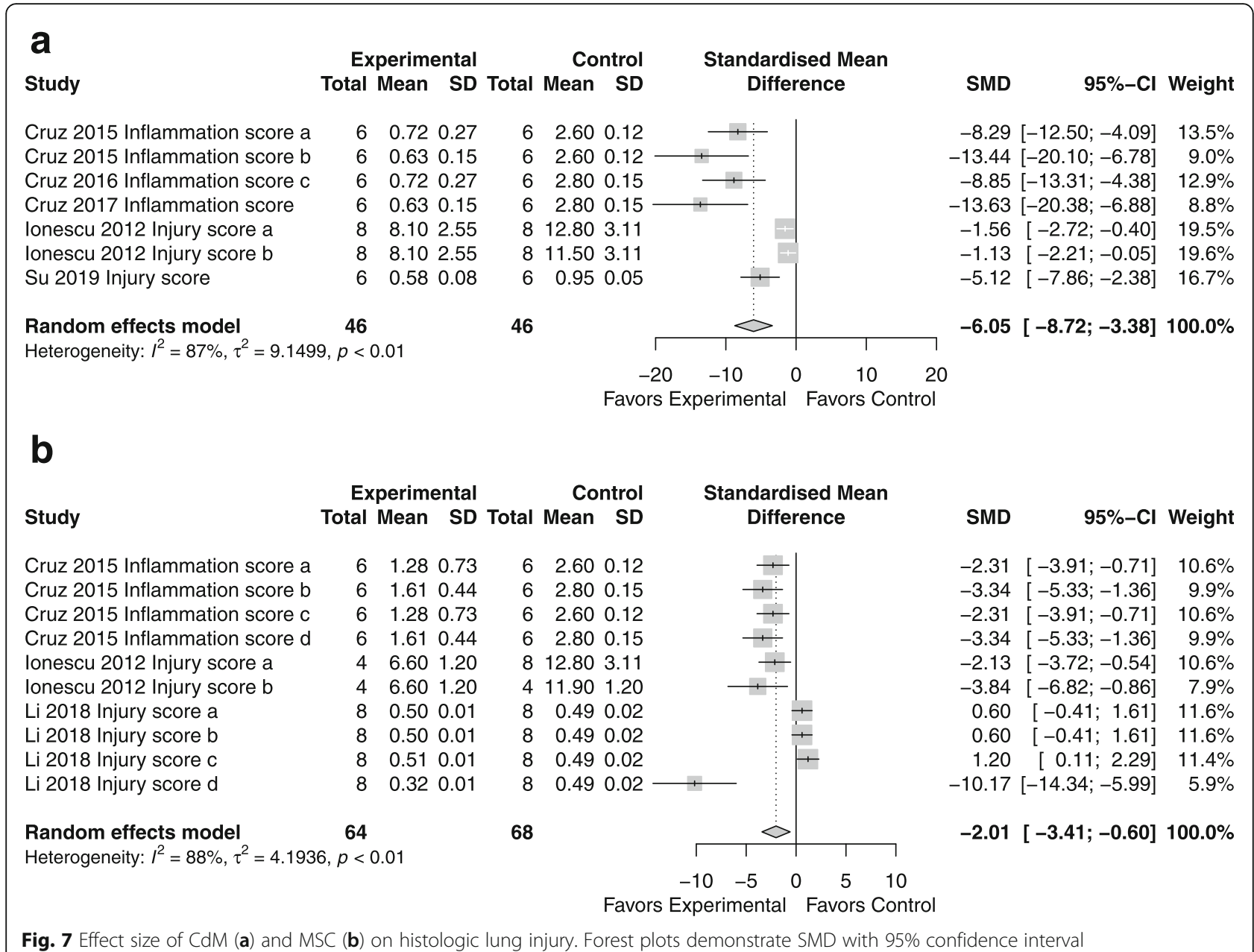

animal models (SMD 1.67) and when the media was derived from cord blood (SMD 2.89), given at a dose of $7 \mu \mathrm{l} / \mathrm{g}$ (SMD 2.89), and delivered via the intraperitoneal route (SMD 1.56).

\section{RVH}

Supplementary Figure 10A-D depicts that CdM significantly improved RVH in BPD animal models (SMD - 0.93) and only when the media was derived from adipose tissue (SMD - 1.05), given at a dose of $100 \mu \mathrm{l}(\mathrm{SMD}-1.14)$ and delivered intravenously $(\mathrm{SMD}-0.86)$.

\section{Fibrosis}

Supplementary Figure 11A-D illustrates that CdM had the greatest impact in animal models of $\mathrm{BPD}$ and $\mathrm{PH}$ (SMD - 4.1, - 3.4, respectively) and when the media was derived from adipose tissue (SMD - 2.61), given at a dose of $50 \mu \mathrm{l}(\mathrm{SMD}-4.10)$ and delivered intravenously (SMD - 1.95).

\section{Vascularization}

Supplementary Figure 12A-D shows that CdM had the greatest impact in animal models of COPD (SMD 8.09 ), when the media was derived from adipose tissue (SMD - 2.61), given at a dose of $300 \mu \mathrm{l}(\mathrm{SMD}-8.09)$ and delivered intravenously (SMD - 3.65).

\section{Risk of bias}

No study was judged as low risk across all ten domains. Eight studies stated that the allocation selection was random. Most studies $(n=25)$ had similar groups at baseline. Risk of bias was large regarding allocation concealment, whether authors mention random housing of animals, and blinding of caregivers or random selection of outcome. All studies were found to sufficiently report complete data and being free from other bias. Refer to Supplementary File 2 [27].

\section{Publication bias}

Supplementary Figures 13, 14, 15, 16, 17, 18, 19, and 20 illustrate publication bias through funnel plots. Overall, 


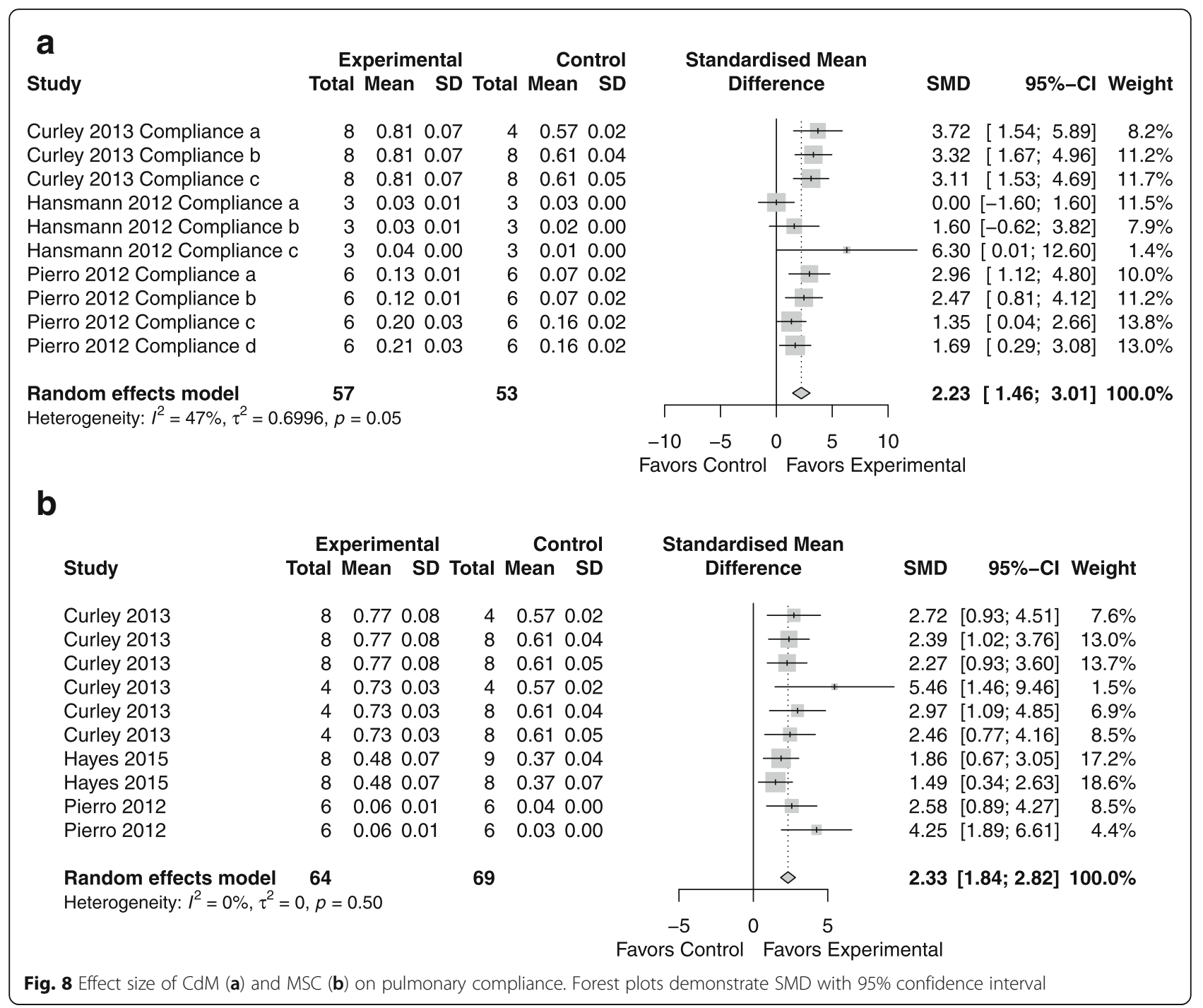

publication bias was low in all the outcomes except for lung permeability.

\section{Discussion}

Preclinical studies reiterate the ability MSCs have on dampening lung inflammation. This capacity is largely due to the paracrine secretion of MSC factors (microvesicles, exosomes) that provide a basis for future cell-free therapies for human disease [28-31]. This is the first review to directly compare the effects of CdM vs MSCs on lung structure and function in animal models of diverse lung disease. Overall, we found that $\mathrm{CdM}$ improved measures of alveolarization, right ventricular hypertrophy, lung fibrosis, vasculogenesis and permeability. Furthermore, CdM reduced pulmonary pressures, ameliorated histologic lung injury, and increased lung compliance. We found that CdM was comparable to MSCs in all lung measures evaluated individually and when combined.

The bioactive factors contained in the CdM of MSCs have been the focus of multiple studies and review articles [32-34]. Congruent with the findings found in this review, Hansmann et al. show that MSC-CdM, compared to $\mathrm{CdM}$ from lung fibroblasts, reversed alveolar injury, normalized lung function (airway resistance), and reversed RVH [35]. Additionally, the same group recently demonstrated that MSC exosomes (molecular cargo found within $\mathrm{CdM}$ ) restored lung architecture, stimulated pulmonary blood vessel formation, and modulated lung inflammation [22]. In an E. coli pneumoniainduced ALI mouse model, MSC microvesicles (also found in MSC-CdM) reduced lung permeability and histologic injury score and were equivalent to MSCs [36]. Together, these findings, and those in recent 
reviews, substantiate the results found in this review [37, 38].

This year, Augustine et al. published a network metaanalysis comparing stem cell and cell-free therapies in preclinical measures of BPD. MSC-CdM had a similar effect size to MSCs regarding alveolarization (MSC SMD 1.71 vs. CdM SMD1.68), angiogenesis (SMD 2.24 vs. 1.79 ), and pulmonary remodeling (1.29 vs. 1.22) [39]. Similar to their results, this review showed that CdM had among the largest impact on measures of alveolarization and vasculogenesis, processes critical for appropriate lung healing, development, and function [40]. Although vasculogenesis/angiogenesis is an important process to restore lung function/structure, it can also enhance remodeling and thus worsen outcomes in other lung diseases such as asthma or pulmonary fibrosis [41]. In Supplementary Figure 12A, we demonstrate that this process improved in BPD, pulmonary hypertension, and COPD but was not assessed in asthma/pulmonary fibrosis.

In the study by Hayes et al., they found that MSCs were superior to $\mathrm{CdM}$ in a rodent model of ventilatorinduced lung injury. However, our review suggests that when you compile the literature, there were no significant benefits of using cells over CdM. We cannot explain why $\mathrm{CdM}$ was not comparable in this study; however, an important challenge that remains in the field includes the rigorous testing of key variables (tissue source, dose, route, disease, etc.) that may impact the quality of CdM [42-44]. For instance, we found that the intravenous route provided optimal results. Moreover, multiple administrations of CdM may augment vascular development, as seen in the study by Huh et al ( $n=10$ intravenous injections). Conversely, the optimal source and dose of $\mathrm{CdM}$ is dependent on the variable or the lung disease. This brings to light that it will be incredibly challenging to find a single $\mathrm{CdM}$ product that is ideal for all lung diseases. Thus, the idea of "one-size-fits-all" does not hold true for regenerative cells or products. Illustrating this concept, Rathinasabapathy et al. showed greater improvement in measures of RVH compared to other studies measuring right ventricular size. Important differences seen in the study by Rathinasabapathy and colleagues was that they used a different animal model (PH vs. BPD) and age of rodents (adult vs. neonatal) [45].

As investigators, we should attempt to tease out these characteristics in order to have the ideal product(s) for our lung disease of interest. In this way, we may have translational success in future clinical studies. Refining these features will take time but will play a vital role in efficacy. Moreover, pinpointing small and large animal models of lung disease that will recapitulate what occurs at the patient bedside is essential if we want to move the needle in the field [46].
The plausibility of using a cell-free product as a therapeutic agent for lung disease is substantiated by newly registered human clinical trials. For instance, NCT04235296 and NCT04234750 are evaluating safety of MSC-CdM in regulating wound inflammation and promoting wound healing in burn injury. Another Phase I trial (NCT04134676) plans to study the therapeutic potential of umbilical cord tissuederived stem cell CdM on chronic skin ulcers. Trials valuing the safety of stem cell CdM constituents (exosomes) are also underway for ischemic stroke (NCT3384433) and ocular conditions (NCT04213248, NCT03437759).

There are several limitations to our systematic review and meta-analysis, many of which mirror those published in our previous report. We incorporated multiple animal models of lung disease that have diverse pathologic processes resulting in their etiology. Also, most of the studies lacked methodologic details rendering them with an unclear risk of bias. Moreover, although preclinical models of lung disease have been helpful in identifying targetable mechanisms/processes, they oftentimes lack the intricacies of human disease. Thus, meticulous efficacy studies in large animals may be one approach to mitigate translational failure in human trials.

\section{Conclusion}

This review demonstrates that the administration of $\mathrm{CdM}$ in animal models of lung disease improves lung architecture and function. When compared to MSCs, $\mathrm{CdM}$ is as efficacious and provides a basis that cell-free products are a viable option for future studies. However, mores studies are needed to identify how specific variables (tissue source, route of delivery, concentration, etc.) may impact/strengthen their therapeutic potential.

\section{Supplementary information}

Supplementary information accompanies this paper at https://doi.org/10. 1186/s13287-020-01900-7.

\section{Additional file 1: Figure S1. Flow diagram demonstrating study} selection process.

Additional file 2: Figure S2. Effect size of CdM vs. MSC on lung alveolarization. . Forest plots demonstrate SMD with 95\% confidence interval.

Additional file 3: Figure S3. Effect size of CdM on right ventricular hypertrophy. Forest plots demonstrate SMD with 95\% confidence interval.

Additional file 4: Figure S4. Effect size of MSC on lung fibrosis. Forest plots demonstrate SMD with 95\% confidence interval.

Additional file 5: Figure S5. Effect size of CdM vs. MSC on pulmonary vasculogenesis. Forest plots demonstrate SMD with 95\% confidence interval.

Additional file 6: Figure S6. Effect size of CdM vs. MSC on lung permeability. Forest plots demonstrate SMD with 95\% confidence interval. 
Additional file 7: Figure S7. Effect size of CdM vs. MSC on pulmonary pressures. Forest plots demonstrate SMD with 95\% confidence interval.

Additional file 8: Figure S8. Effect size of CdM (a), MSCs (b), and CdM vs. MSC (c) on all eight outcomes. Forest plots demonstrate SMD with 95\% confidence interval.

Additional file 9: Figure S9. Effect size of $\mathrm{CdM}$ on lung alveolarization by disease (a), source (b), dose (c), and route (d). Forest plots demonstrate SMD with 95\% confidence interval.

Additional file 10: Figure S10. Effect size of $\mathrm{CdM}$ on right ventricular hypertrophy by disease (a), source (b), dose (c), and route (d). Forest plots demonstrate SMD with 95\% confidence interval.

Additional file 11: Figure S11. Effect size of $\mathrm{CdM}$ on lung fibrosis by disease (a), source (b), dose (c), and route (d). Forest plots demonstrate SMD with 95\% confidence interval.

Additional file 12: Figure S12. Effect size of $\mathrm{CdM}$ on pulmonary vascularization by disease (a), source (b), dose (c), and route (d). Forest plots demonstrate SMD with 95\% confidence interval.

Additional file 13: Figure S13. Funnel plot assessing for publication bias of $\mathrm{CdM}$ on lung alveolarization.

Additional file 14: Figure S14. Funnel plot assessing for publication bias of CdM on right ventricular hypertrophy.

Additional file 15: Figure S15. Funnel plot assessing for publication bias of $\mathrm{CdM}$ on lung fibrosis.

Additional file 16: Figure S16. Funnel plot assessing for publication bias of $\mathrm{CdM}$ on pulmonary vasculogenesis.

Additional file 17: Figure S17. Funnel plot assessing for publication bias of CdM on lung permeability.

Additional file 18: Figure S18. Funnel plot assessing for publication bias of $\mathrm{CdM}$ on pulmonary pressures.

Additional file 19: Figure S19. Funnel plot assessing for publication bias of CdM on histologic lung injury.

Additional file 20: Figure S20. Funnel plot assessing for publication bias of CdM on lung compliance.

Additional file 21: File S1. List of articles included in this review. Additional file 22: File S2. SYRCLE risk of bias.

Additional file 23: File S3. CdM characteristics.

\section{Abbreviations}

ARDS: Acute respiratory distress syndrome; BPD: Bronchopulmonary dysplasia; CAMARADES: Collaborative Approach to Meta-Analysis and Review of Data from Experimental Studies; CdM: Conditioned media; CF: Cystic fibrosis; Cl: Confidence interval; MSC: Mesenchymal stem/stromal cell; NCT: National clinical trial; PH: Pulmonary hypertension; SMD: Standardized mean difference; SYRCLE: Systematic Review Centre for Laboratory Animal Experimentation

\section{Acknowledgements}

Dr. Robert Cote, Director of the Writing Skills Program at the University of Arizona, Tucson.

\section{Authors' contributions}

AM contributed to the design of review, analysis of data, manuscript data, and oversight. RN contributed to the data collection, manuscript writing, and risk of bias. $\mathrm{KH}$ contributed to the data collection and manuscript writing. CE contributed to the database search, design of review, assembly of data, and analysis of data. JM contributed to the data collection. AM contributed to the design of review, manuscript writing, and interpretation of data. ED contributed to the database search, design of review, and data collection. SZ contributed to the manuscript writing and interpretation of data. ME contributed to the data collection, risk of bias, and Tables 1 and 2. DM contributed to the data collection and Tables 1 and 2. SM contributed to the data collection and Tables 1 and 2 . The author(s) read and approved the final manuscript.

\section{Funding}

Supported by NIH NCATS KL2 TR001118, UT Health San Antonio School of Medicine pilot grant, Parker B. Francis Foundation (provided to senior author).

Availability of data and materials

Availability of data and materials will be available through figshare upon publication of the manuscripts.

Ethics approval and consent to participate

Not applicable.

\section{Consent for publication}

All authors have looked through the manuscript and approved the submission

\section{Competing interests}

The authors declare that they have no competing interests.

\section{Author details}

'Department of Pediatrics, Division of Neonatology, University of Texas Health Science-San Antonio, San Antonio, TX 78229-3900, USA. ${ }^{2}$ Department of Pediatrics, Division of Critical Care, Baylor College of Medicine, Houston, TX, USA.

Received: 12 February 2020 Revised: 19 August 2020 Accepted: 24 August 2020 Published online: 15 September 2020

\section{References}

1. Ferkol T, Schraufnagel D. The global burden of respiratory disease. Ann Am Thorac Soc. 2014;11:404-6. Available from: http://www.atsjournals.org/doi/ abs/10.1513/AnnalsATS.201311-405PS. [cited 2019 Sep 30].

2. Winterstein AG, Choi Y, Cody MH. Association of age with risk of hospitalization for respiratory syncytial virus in preterm infants with chronic lung disease. JAMA Pediatr. 2018;172:154-60.

3. Maxwell BG, Nies MK, Ajuba-Iwuji CC, Coulson JD, Romer LH. Trends in hospitalization for pediatric pulmonary hypertension. Pediatrics. 2015:136: 241-50.

4. Bui DS, Lodge CJ, Burgess JA, Lowe AJ, Perret J, Bui MQ, et al. Childhood predictors of lung function trajectories and future COPD risk: a prospective cohort study from the first to the sixth decade of life. Lancet Respir Med. 2018:6:535-44

5. Savran O, Ulrik CS. Early life insults as determinants of chronic obstructive pulmonary disease in adult life. Int J Chron Obstruct Pulmon Dis. 2018:13: 683-93. https://doi.org/10.2147/COPD.S153555. Published 2018 Feb 26.

6. Zhou-Suckow Z, Duerr J, Hagner M, Agrawal R, Mall MA. Airway mucus, inflammation and remodeling: emerging links in the pathogenesis of chronic lung diseases. Cell Tissue Res. 2017:367(3):537-50.

7. Virk $H$, Arthur $G$, Bradding P. Mast cells and their activation in lung disease. Transl Res. 2016:174:60-76.

8. Barnes PJ. Cellular and molecular mechanisms of asthma and COPD. Clin Sci (Lond). 2017:131(13):1541-58.

9. Robinson D, Humbert M, Buhl R, et al. Revisiting Type 2-high and Type 2low airway inflammation in asthma: current knowledge and therapeutic implications. Clin Exp Allergy. 2017:47(2):161-75.

10. Becerra-Díaz M, Wills-Karp M, Heller NM. New perspectives on the regulation of type II inflammation in asthma. F1000Research. 2017:6:1014. Available from: http://www.ncbi.nlm.nih.gov/pubmed/28721208. [cited 2019 Jun 11].

11. Dias-Freitas F, Metelo-Coimbra C, Roncon-Albuquerque R Jr. Molecular mechanisms underlying hyperoxia acute lung injury. Respir Med. 2016;119: 23-8.

12. Barsky EE, Giancola LM, Baxi SN, Gaffin JM. A Practical Approach to Severe Asthma in Children [published correction appears in Ann Am Thorac Soc. 2018;15(6):767-8.

13. Curley GF, Hayes M, Ansari B, Shaw G, Ryan A, Barry F, et al. Mesenchymal stem cells enhance recovery and repair following ventilator-induced lung injury in the rat. Thorax. 2012;67:496-501. Available from: http://www.ncbi. nlm.nih.gov/pubmed/22106021. [cited 2017 Dec 16].

14. Zhong $H$, Fan XL, Fang S, Bin LYD, Wen W, Fu QL. Human pluripotent stem cell-derived mesenchymal stem cells prevent chronic allergic airway 
inflammation via TGF- $\beta 1$-Smad2/Smad3 signaling pathway in mice. Mol Immunol. 2019;109:51-7.

15. Cui $P$, Xin $H, Y a o ~ Y$, et al. Human amnion-derived mesenchymal stem cells alleviate lung injury induced by white smoke inhalation in rats. Stem Cell Res Ther. 2018;9(1):101.

16. Phinney DG, Pittenger MF. Concise review: MSC-derived exosomes for cell free therapy. Stem Cells. 2017;35:851-8.

17. Vizoso FJ, Eiro N, Cid S, Schneider J, Perez-Fernandez R. Mesenchymal stem cell secretome: toward cell-free therapeutic strategies in regenerative medicine. Int J Mol Sci. 2017;18 Available from: http://www.ncbi.nlm.nih. gov/pubmed/28841158. [cited 2017 Dec 16].

18. Moreira A, Kahlenberg S, Hornsby P. Therapeutic potential of mesenchymal stem cells for diabetes. J Mol Endocrinol. 2017;59:R109-20. Available from: http://www.ncbi.nlm.nih.gov/pubmed/28739632. [cited 2017 Nov 30].

19. Zakrzewski W, Dobrzyński M, Szymonowicz M, Rybak Z. Stem cells: past, present, and future. Stem Cell Res Ther. 2019;10(1):68.

20. Cruz FF, PRM R. Hypoxic preconditioning enhances mesenchymal stromal cell lung repair capacity. Stem Cell Res Ther. 2015;6:130. Available from: http://www.ncbi.nlm.nih.gov/pubmed/26169784. [cited 2017 Nov 16].

21. Ferreira JR, Teixeira GQ, Santos SG, Barbosa MA, Almeida-Porada G, Gonçalves RM. Mesenchymal stromal cell secretome: influencing therapeutic potential by cellular pre-conditioning. Front Immunol. 2018;9: 2837.

22. Willis GR, Fernandez-Gonzalez A, Anastas J, Vitali SH, Liu X, Ericsson M, et al. Mesenchymal stromal cell exosomes ameliorate experimental bronchopulmonary dysplasia and restore lung function through macrophage immunomodulation. Am J Respir Crit Care Med. 2018;197:10416. Available from: http://www.ncbi.nlm.nih.gov/pubmed/28853608. [cited 2019 Jun 21].

23. van Haaften T, Byrne R, Bonnet S, Rochefort GY, Akabutu J, Bouchentouf M, et al. Airway delivery of mesenchymal stem cells prevents arrested alveolar growth in neonatal lung injury in rats. Am J Respir Crit Care Med. 2009:180: 1131-42. Available from: http://www.ncbi.nlm.nih.gov/pubmed/19713449. [cited 2016 Oct 27].

24. Emukah C, Dittmar E, Naqvi R, et al. Mesenchymal stromal cell conditioned media for lung disease: a systematic review and meta-analysis of preclinical studies. Respir Res. 2019;20(1):239.

25. Zeng X, Zhang Y, Kwong JS, et al. The methodological quality assessment tools for preclinical and clinical studies, systematic review and metaanalysis, and clinical practice guideline: a systematic review. J Evid Based Med. 2015; 8(1):2-10.

26. Welcome to CAMARADES. Available from: http://www.den.ed.ac.uk/ camarades/. [cited 2020 Jan 30].

27. Hooijmans CR, Rovers MM, De Vries RBM, Leenaars M, Ritskes-hoitinga M, Langendam MW. SYRCLE's risk of bias tool for animal studies. BMC Med Res Methodol. 2014;14:1-9.

28. Bruno S, Kholia S, Deregibus MC, Camussi G. The role of extracellular vesicles as paracrine effectors in stem cell-based therapies. Adv Exp Med Biol. 2019;1201:175-93.

29. Joo HS, Suh JH, Lee HJ, Bang ES, Lee JM. Current knowledge and future perspectives on mesenchymal stem cell-derived exosomes as a new therapeutic agent. Int J Mol Sci. 2020;21(3):727. Published 2020 Jan 22. https://doi.org/10.3390/ijms21030727.

30. Liu C, Wang J, Hu J, Fu B, Mao Z, Zhang H, et al. Extracellular vesicles for acute kidney injury in preclinical rodent models: a meta-analysis. Stem Cell Res Ther. 2020;11:11.

31. Willis GR, Mitsialis SA, Kourembanas S. "Good things come in small packages": application of exosome-based therapeutics in neonatal lung injury. Pediatr Res. 2018:83:298-307. Available from: http://www.nature.com/ articles/pr2017256. [cited 2019 Jul 12].

32. Madrigal M, Rao KS, Riordan NH. A review of therapeutic effects of mesenchymal stem cell secretions and induction of secretory modification by different culture methods. J Transl Med. 2014;12:260.

33. Pawitan JA. Prospect of stem cell conditioned medium in regenerative medicine. Biomed Res Int. 2014;2014:965849. Available from: http://www. ncbi.nlm.nih.gov/pubmed/25530971. [cited 2019 Jul 20].

34. Augustine S, Avey MT, Harrison B, Locke T, Ghannad M, Moher D, et al. Mesenchymal stromal cell therapy in bronchopulmonary dysplasia: Systematic review and meta-analysis of preclinical studies. Stem Cells Transl Med. 2017;6:2079-93. Available from: http://www.ncbi.nlm.nih.gov/ pubmed/29045045. [cited 2019 Mar 22].
35. Hansmann G, Fernandez-Gonzalez A, Aslam M, Vitali SH, Martin T, Mitsialis $S A$, et al. Mesenchymal stem cell-mediated reversal of bronchopulmonary dysplasia and associated pulmonary hypertension. Pulm Circ. 2012;2:170-81. Available from: http://www.ncbi.nlm.nih.gov/pubmed/22837858. [cited 2018 Jan 18].

36. Zhu YG, Feng XM, Abbott J, Fang XH, Hao Q, Monsel A, et al. Human mesenchymal stem cell microvesicles for treatment of Escherichia coli endotoxin-induced acute lung injury in mice. Stem Cells. 2014;32:116-25.

37. Lanyu Z, Feilong $\mathrm{H}$. Emerging role of extracellular vesicles in lung injury and inflammation. Biomed Pharmacother. 2019;113:108748.

38. Fujita Y, Kadota T, Araya J, Ochiya T, Kuwano K. Clinical application of mesenchymal stem cell-derived extracellular vesicle-based therapeutics for inflammatory lung diseases. J Clin Med. 2018;7:355.

39. Augustine S, Cheng W, Avey MT, Chan ML, Lingappa SMC, Hutton B, et al. Are all stem cells equal? Systematic review, evidence map, and metaanalyses of preclinical stem cell-based therapies for bronchopulmonary dysplasia. Stem Cells Transl Med. 2020;9:158-68. Available from: https:// onlinelibrary.wiley.com/doi/abs/10.1002/sctm.19-0193. [cited 2020 Jan 30].

40. Alvira CM. Aberrant pulmonary vascular growth and remodeling in bronchopulmonary dysplasia. Front Med. 2016;3:21. Available from: http:// journal.frontiersin.org/Article/10.3389/fmed.2016.00021/abstract. [cited 2018 Nov 8].

41. Kropski J, Richmond B, Gaskill C, Foronjy R, Majika S. Deregulated angiogenesis in chronic lung diseases: a possible role for lung mesenchymal progenitor cells (2017 Grover Conference Series). Pulm Circ. 2018:8:2045893217739807.

42. Sensebé L, Gadelorge M, Fleury-Cappellesso S. Production of mesenchymal stromal/stem cells according to good manufacturing practices: a review. Stem Cell Res Ther. 2013;4:66. Available from: http://www.ncbi.nlm.nih.gov/ pubmed/23751270. [cited 2017 Jul 11].

43. Galipeau J, Sensébé L. Mesenchymal stromal cells: clinical challenges and therapeutic opportunities. Cell Stem Cell. 2018;22(6):824-33.

44. Lopes-Pacheco M, Robba C, Rocco P. Pelosi P. Current understanding of the therapeutic benefits of mesenchymal stem cells in acute respiratory distress syndrome. Cell Biol Toxicol. 2020;36:83-102.

45. Rathinasabapathy A, Bruce E, Espejo A, et al. Therapeutic potential of adipose stem cell-derived conditioned medium against pulmonary hypertension and lung fibrosis. Br J Pharmacol. 2016;173(19):2859-79. https://doi.org/10.1111/bph.13562.

46. Chamuleau SAJ, van der Naald M, Climent AM, Kraaijeveld AO, Wever KE, Duncker DJ, et al. Translational research in cardiovascular repair. Circ Res. 2018;122:310-8. Available from: http://www.ncbi.nlm.nih.gov/pubmed/2934 8252. [cited 2019 may 3].

\section{Publisher's Note}

Springer Nature remains neutral with regard to jurisdictional claims in published maps and institutional affiliations. 